\title{
Paper Product of Three Random Variables and its Application in Relay Telecommunication Systems in the Presence of Multipath Fading
}

\author{
Dragana Krstic ${ }^{1}$, Petar Nikolic ${ }^{2}$, Danijela Aleksic ${ }^{3}$, Sinisa Minic ${ }^{4}$, \\ Dragan Vuckovic ${ }^{5}$, and Mihajlo Stefanovic ${ }^{1}$ \\ ${ }^{1}$ Faculty of Electronic Engineering, University of Niš, Niš, Serbia \\ 2 TigarTyres, Pirot, Serbia \\ ${ }^{3}$ College of Applied Technical Sciences Nišs, Serbia \\ ${ }^{4}$ Teachers' Training Faculty, Prizren-Leposavic, University of Pristina, Kosovska Mitrovica, Serbia \\ ${ }^{5}$ Faculty of Economics and Engineering Management, University Business Academy, Novi Sad, Serbia
}

https://doi.org/10.26636/jtit.2019.130018

\begin{abstract}
In this paper, the product of three random variables (RVs) will be considered. Distribution of the product of independent random variables is very important in many applied problems, including wireless relay telecommunication systems. A few of such products of three random variables are observed in this work: the level crossing rate (LCR) of the product of a Nakagami- $m$ random variable, a Rician random variable and a Rayleigh random variable, and of the products of two Rician RVs and one Nakagami-m RV is calculated in closed forms and presented graphically. The LCR formula may be later used for derivation of average fade duration (AFD) of a wireless relay communication radio system with three sections, working in the multipath fading channel. The impact of fading parameters and multipath fading power on the LCR is analyzed based on the graphs presented.
\end{abstract}

Keywords-level crossing rate, Nakagami-m fading, Rayleigh fading, relay telecommunication systems, Rician fading.

\section{Introduction}

Statistical characteristics of products and ratios of random variables (RVs) are essential in analyzing the performance of contemporary wireless telecommunications systems, as well as in solving numerous applied problems. The products of RVs are encountered naturally in such applications as: channel modeling, multihop wireless relaying systems, cascaded fading channels, MIMO keyhole systems [1], quantum physics, signal processing, tensor sensing problem, the rate offset of the hybrid automatic repeat request (H-ARQ) transmission, and even in biological and physical sciences, econometrics, classification, ranking and selection [2].

Because of that, in recent years, the products and ratios of random processes are investigated in the literature by many researchers [1]-[6]. In the 1960s, Donahue, Springer,
Thompson and Lomnicki started with derivations concerning the distributions of the products of two RVs [7]-[10]. At the beginning of this century, interest in this area has increased again [11] - [13]. The latest works, with significant application in wireless communications systems, are [14]-[17].

Computational algorithms for derivating the distribution of the product of two RVs are given in [9]. The product and the ratio of two independent, Student's t distributed RVs, are observed in [10]. The derivation of the probability density function (PDF) of the product of two independent, non-identical, and triangularly distributed RVs, by using integral calculation, is presented in [11].

The problem of characterizing products of independent $\mathrm{RVs}$ is investigated for normal RVs, products of their absolute values, and products of their squares in [13]. Power$\log$ series expansions of cumulative distribution functions (CDF), based on the theory of Fox $\mathrm{H}$ functions, is computed. It is numerically shown that CDF is well approximated by the lowest orders of this expansion for small arguments. The moment generating functions (MGF) in terms of Meijer $G$ functions are also computed for two non-negative RVs. In that paper, the fading amplitudes of cascaded fading channels have the distribution of the product of Nakagami- $m$ RVs, as in [5] and [6], and of the product of Rician RVs, as in [18] and [19].

Signal envelope variations, called fading, are results of reflections, refractions, diffraction and scattering. They can be described by several distributions. So, Rayleigh [20] and Nakagami- $m$ [21] distributions are used when no dominant component is present. Signal envelope variation is modeled by Rician distribution when a line-of-sight (LOS) dominant component exists in the channel [22].

Level crossing rate (LCR) of a product of two Nakagami- $m$ random processes is analyzed in [6]. Then, the av- 
erage fade duration (AFD) of a wireless relay communications system consisting of two sections in a Nakagami- $m$ short term fading channel is obtained. The performance of the product of arbitrary and independent RVs with a general $\alpha-\mu$ distribution is given in [15]. The closed-form expressions for PDF, CDF and moments are obtained and the calculation process used to obtain the amount of fading (AoF) and outage probability (OP) in cascaded channels is presented.

An analysis of the performance of the product of two independent and non-identically distributed $\kappa-\mu \mathrm{RVs}$ is presented in [17], where analytical formulations for PDF, $\mathrm{CDF}$ and MGF are obtained. By using these formulations, closed-form expressions for higher order moments, AoF and channel quality estimation index are derived, as are analytical formulas for OP, average channel capacity, average symbol error probability (SEP) and average bit error probability. This applies to different fading scenarios, such as double Rayleigh, double Rician, double Nakagami- $m, \kappa-\mu /$ Nakagami- $m$, and Rician/Nakagami- $m$, identified as special cases.

The cascaded keyhole channels may be modeled using the product of individual channels [23]. Further, independent and identically distributed (i.i.d.) double fading channels in a line-of-sight (LOS) environment, typical for keyhole Multiple input multiple output (MIMO) systems, are analyzed in [24]. LOS double fading, i.e. double Rician fading in MIMO channels, is investigated and the density function of the signal-to-noise ratio (SNR) is discussed. After obtaining the exact expression in a form with infinite series, an approximation formula of SNR density is presented by using the Nakagami- $m$ approximation of Rician distribution.

In [25], the product of Nakagami-m RV, Rician RV and Rayleigh RV is analyzed. LCR of this product is calculated. The results obtained may also be used for the evaluation the AFD of a relay wireless communications system with three sections in the presence of Nakagami- $m$ fading in the first section, Rician fading in the second section and Rayleigh fading in the third section. LCR of the product of three independent Rician RVs is observed in [26].

In this article, except for the results from [25], the product of two Rician RVs and one Nakagami- $m$ RV is processed. The result can be applied for derivation, in a closed form, of the second order performance of a wireless relay communications system with three sections operating in Rician and Nakagami- $m$ fading environments. The formulas are validated by numerical results and impact of the individual parameters is analyzed.

This work is composed of four sections. Section 1 serves as an introduction and describes previous works in the area. In two next sections, the product of three random variables is given and an expression for LCR in a closed form is performed for two different sets of RVs. The influence of parameters is shown via several graphics for both sets. The work ends with conclusions presented in Section 4.

\section{Derivation of LCR of Product of Nakagami- $m$, Rician and Rayleigh Random Variables}

Here, we examine the scenario involving a wireless relay communications system with three sections. The signal envelope at the output of the relay communications system with three sections is the product of envelopes at the individual sections. In the first example observed, the Nakagami- $m$ signal envelope is at the first section, the Rician signal envelope at the second section and the Rayleigh signal envelope at the third section.

As a rule, it is first necessary to calculate the probability density function. By using PDF, bit error probability (BEP) can be evaluated, and by using CDF, outage probability can be obtained [27], [28]. OP and BEP are the first order performance measure of the wireless communications system. OP may be calculated as probability that the signal envelope is below the threshold [27], [29]. Level crossing rate is the second order statistic measure of the wireless communications system and is associated with envelope fading, as well as with average fade duration. LCR shows how often the envelope crosses a specified level and may be calculated as the number of crossings at this defined level. AFD shows how long the envelope remains below a specified level and can be evaluated as the ratio of OP and LCR. These two quantities are second order statistics because they are affected not only by the scattering in environment, but also by the speed of mobile stations. Here, PDF of the product of Nakagami- $m$ RV, Rician RV and Rayleigh RV will be obtained by using the transformation method. Further, using this PDF, CDF and moments can be evaluated, as can be the level crossing rate.

\subsection{Distribution of Random Variables}

Nakagami- $m$ random variable $x_{1}$ follows the distribution defined in [21]:

$$
p_{x_{1}}\left(x_{1}\right)=\frac{2}{\Gamma(m)}\left(\frac{m}{\Omega_{1}}\right)^{m} x_{1}^{2 m-1} e^{-\frac{m}{\Omega_{1}} x_{1}^{2}}, m \geq \frac{1}{2}, x_{1} \geq 0
$$

where $\Gamma($.$) is a gamma function. This distribution has two$ parameters. The first parameter controls spread. Actually, $\Omega_{1}=E\left[x_{1}^{2}\right]$ is the average power of the multipath scattering field. $m$ is the fading depth parameter or the shape factor of the Nakagami distribution [30]. For RV $x_{1}$ the shape parameter is:

$$
m=\frac{\Omega_{1}^{2}}{E\left\{\left[x_{1}^{2}-\Omega_{1}^{2}\right]^{2}\right\}} .
$$

It describes the fading degree of the propagation environment caused by the interference of scattering and multipath phenomena. So, the severity of fading is indicated by the Nakagami parameter $m$. 
Random variable $x_{2}$ follows Rician distribution [22]:

$$
\begin{aligned}
p_{x_{2}}\left(x_{2}\right)=\frac{2(\kappa+1)}{\Omega_{2}} & \sum_{i_{1}=0}^{\infty}\left(\frac{\kappa(\kappa+1)}{\Omega_{2}}\right)^{i_{1}} \\
& \times \frac{1}{\left(i_{1} !\right)^{2}} x_{2}^{2 i_{1}+1} \mathrm{e}^{-\frac{\kappa+1}{\Omega_{2}} x_{2}^{2}}, \quad x_{2} \geq 0
\end{aligned}
$$

where $\Omega_{2}$ is the average received power for $x_{2}$ and $\kappa$ is the Rician factor. Rician factor $\kappa$ is defined as a ratio of dominant component's power and the scattering components' powers. This type of fading, called Rician fading, is very often observed in microcellular and mobile satellite applications [27].

Rayleigh distribution can be easily derived from Rician distribution for Rician factor $\kappa=0$. For $\kappa=\infty$ we have no fading, i.e. a channel with no multipath and only a LOS component. The fading parameter $\kappa$ is therefore a measure of the severity of fading: a small $\kappa$ implies severe fading, a large $\kappa$ implies more mild fading [31].

Two-dimensional isotropic scattering, where the arriving waves arrive to the receiver from all directions, with equal probability, is a scattering model that is often used for the communication channel in a macrocellular system. For this type of scattering environment, the received envelope is Rayleigh distributed at any time, and is said to be Rayleigh fading.

Random variable $x_{3}$ follows Rayleigh distribution [31]:

$$
p_{x_{3}}\left(x_{3}\right)=\frac{x_{3}}{\Omega_{3}} \mathrm{e}^{-\frac{x_{3}^{3}}{\Omega_{3}}}, \quad x_{3} \geq 0,
$$

where $\Omega_{3}$ is the average received signal power of signal $x_{3}$, i.e. the received power based alone on path loss and shadowing alone [31].

\subsection{Product of Three Random Variables}

The product of three random variables $x_{1}, x_{2}$ and $x_{3}$ is:

$$
x=x_{1} x_{2} x_{3}
$$

Then, it is valid that:

$$
x_{1}=\frac{x}{x_{2} x_{3}} .
$$

The first time derivative of $x$ is:

$$
\dot{x}=\dot{x}_{1} x_{2} x_{3}+x_{1} \dot{x}_{2} x_{3}+x_{1} x_{2} \dot{x}_{3} .
$$

The first time derivative of $x_{i}$ has Gaussian distribution:

$$
p_{\dot{x}_{i}}\left(\dot{x}_{i}\right)=\frac{1}{\sqrt{2 \pi \dot{\sigma}_{i}^{2}}} \mathrm{e}^{-\frac{\dot{x}_{i}^{2}}{2 \dot{\sigma}_{i}^{2}}}, \quad-\infty<\dot{x}_{1}<\infty,
$$

where $\dot{\sigma}_{1}^{2}=\frac{\pi^{2} f_{m}^{2} \Omega_{i}}{m}$ and $f_{m}$ being maximal Doppler frequency. The processes $x_{i}$ and $\dot{x}_{i}$ are considered to be independent, as Rice demonstrated in [32].
This is a very interesting result which shows that, in the Nakagami case and in the Rayleigh and Rice cases, $x_{i}$ and $\dot{x}_{i}$ are mutually independent random variables [33], i.e., it is valid that [32]:

$$
p_{x_{i} \dot{x}_{i}}\left(x_{i} \dot{x}_{i}\right)=p_{x_{i}}\left(x_{i}\right) p_{\dot{x}_{i}}\left(\dot{x}_{i}\right) \text {. }
$$

Moreover, the probability density function of the time derivative of the Nakagami envelope is also Gaussian distributed as are the time derivatives of both Rayleigh and Rice envelopes [34].

So, all random variables $\dot{x}_{1}, \dot{x}_{2}$, and $\dot{x}_{3}$ have Gaussian distribution. A linear combination of Gaussian RVs is a Gaussian RV. The mean signal level of $\dot{x}$ is:

$$
\overline{\dot{x}}=\overline{\dot{x}}_{1} x_{2} x_{3}+x_{1} \overline{\dot{x}}_{2} x_{3}+x_{1} x_{2} \overline{\dot{x}}_{3}=0
$$

because:

$$
\overline{\dot{x}}_{1}=\overline{\dot{x}}_{2}=\overline{\dot{x}}_{3}=0 \text {. }
$$

The variance of $\dot{x}$ is:

$$
\sigma_{\dot{x}}^{2}=x_{2}^{2} x_{3}^{2} \sigma_{\dot{x}_{1}}+x_{1}^{2} x_{3}^{2} \sigma_{\dot{x}_{2}}+x_{1}^{2} x_{2}^{2} \sigma_{\dot{x}_{3}}
$$

where:

$$
\begin{aligned}
& \sigma_{\dot{x}_{1}}=\pi^{2} f_{m}^{2} \frac{\Omega_{1}}{m}, \\
& \sigma_{\dot{x}_{2}}=\pi^{2} f_{m}^{2} \frac{\Omega_{2}}{\kappa+1}, \\
& \sigma_{\dot{x}_{3}}=\pi^{2} f_{m}^{2} \Omega_{3} .
\end{aligned}
$$

After substituting, the expression for variance becomes:

$$
\begin{aligned}
& \sigma_{\dot{x}}^{2}=\pi^{2} f_{m}^{2}\left(x_{2}^{2} x_{3}^{2} \frac{\Omega_{1}}{m}+x_{1}^{2} x_{3}^{2} \frac{\Omega_{2}}{\kappa+1}+x_{1}^{2} x_{2}^{2} \Omega_{3}\right) \\
& =\pi^{2} f_{m}^{2} x_{2}^{2} x_{3}^{2} \frac{\Omega_{1}}{m}\left(1+\frac{x^{2}}{x_{2}^{4} x_{3}^{2}} \frac{\Omega_{2}}{\Omega_{1}} \frac{m}{\kappa+1}+\frac{x^{2}}{x_{2}^{2} x_{3}^{4}} \frac{\Omega_{3}}{\Omega_{1}} m\right) .
\end{aligned}
$$

The joint probability density function of $x, \dot{x}, x_{2}$ and $x_{3}$ is: $p_{x \dot{x} x_{2} x_{3}}\left(x \dot{x} x_{2} x_{3}\right)=p_{\dot{x}}\left(\dot{x} / x x_{2} x_{3}\right) p_{x}\left(x / x_{2} x_{3}\right) p_{x_{2}}\left(x_{2}\right) p_{x_{3}}\left(x_{3}\right),($

where

$$
\begin{gathered}
p_{x}\left(x / x_{2} x_{3}\right)=\left|\frac{d x_{1}}{d x}\right| p_{x_{1}}\left(\frac{x}{x_{2} x_{3}}\right), \\
\frac{d x_{1}}{d x}=\frac{1}{x_{2} x_{3}} .
\end{gathered}
$$

The joint probability density function of $x$ and $\dot{x}$ is:

$$
\begin{aligned}
p_{x \dot{x}}(x \dot{x})=\int_{0}^{\infty} \mathrm{d} x_{2} \int_{0}^{\infty} \mathrm{d} x_{3} p_{\dot{x}}\left(\dot{x} / x x_{2} x_{3}\right) \frac{1}{x_{2} x_{3}} \\
\quad \times p_{x_{1}}\left(\frac{x}{x_{2} x_{3}}\right) p_{x_{2}}\left(x_{2}\right) p_{x_{3}}\left(x_{3}\right) .
\end{aligned}
$$




\subsection{LCR of Product of Three Random Variables}

Level crossing rate of $x$ in a fading environment is [35]:

$$
N_{x}=\int_{0}^{\infty} \mathrm{d} \dot{x} \dot{x} p_{x \dot{x}}(x \dot{x}) .
$$

For our case LCR is:

$$
\begin{aligned}
& N_{x}=\int_{0}^{\infty} \mathrm{d} x_{2} \int_{0}^{\infty} \mathrm{d} x_{3} \frac{1}{x_{2} x_{3}} p_{x_{1}}\left(\frac{x}{x_{2} x_{3}}\right) p_{x_{2}}\left(x_{2}\right) p_{x_{3}}\left(x_{3}\right) \\
& \times \int_{0}^{\infty} \mathrm{d} \dot{x} \dot{x} p_{\dot{x}}\left(\dot{x} / x x_{2} x_{3}\right)=\int_{0}^{\infty} \mathrm{d} x_{2} \int_{0}^{\infty} \mathrm{d} x_{3} \frac{1}{x_{2} x_{3}} p_{x_{1}}\left(\frac{x}{x_{2} x_{3}}\right) p_{x_{2}}\left(x_{2}\right) \\
& \times p_{x_{3}}\left(x_{3}\right) \frac{1}{\sqrt{2 \pi}} \sigma_{\dot{x}}=\int_{0}^{\infty} \mathrm{d} x_{2} \int_{0}^{\infty} \mathrm{d} x_{3} \frac{1}{x_{2} x_{3}} p_{x_{1}}\left(\frac{x}{x_{2} x_{3}}\right) p_{x_{2}}\left(x_{2}\right) \\
& \times p_{x_{3}}\left(x_{3}\right) \frac{1}{\sqrt{2 \pi}} \pi f_{m} x_{2} x_{2} \frac{\Omega^{\frac{1}{2}}}{m^{\frac{1}{2}}}\left(1+\frac{x^{2}}{x_{2}^{4} x_{3}^{2}} \frac{\Omega_{2}}{\Omega_{1}} \frac{m}{\kappa+1}\right. \\
& \left.+\frac{x^{2}}{x_{2}^{2} x_{3}^{4}} \frac{\Omega_{3}}{\Omega_{1}} m\right)^{\frac{1}{2}}=\frac{1}{\sqrt{2 \pi}} \pi f_{m} \frac{\Omega_{1}^{\frac{1}{2}}}{m^{\frac{1}{2}}} \int_{0}^{\infty} \mathrm{d} x_{2} \int_{0}^{\infty} \mathrm{d} x_{3} \\
& \times p_{x_{1}}\left(\frac{x}{x_{2} x_{3}}\right) p_{x_{2}}\left(x_{2}\right) p_{x_{3}}\left(x_{3}\right)\left(1+\frac{x^{2}}{x_{2}^{4} x_{3}^{2}} \frac{\Omega_{2}}{\Omega_{1}} \frac{m}{\kappa+1}\right. \\
& \left.+\frac{x^{2}}{x_{2}^{2} x_{3}^{4}} \frac{\Omega_{3}}{\Omega_{1}} m\right)^{\frac{1}{2}}=\frac{1}{\sqrt{2 \pi}} \pi f_{m} \frac{\Omega_{1}^{\frac{1}{2}}}{m^{\frac{1}{2}}} \frac{2}{\Gamma(m)}\left(\frac{m}{\Omega_{1}}\right)^{m} \\
& \quad \times x^{2 m-1} \frac{2(\kappa+1)}{\Omega_{2}} \sum_{i_{1}=0}^{\infty}\left(\frac{\kappa(\kappa+1)}{\Omega_{2}}\right)^{i_{1}} \frac{1}{\left(i_{1} !\right)^{2}} \frac{2}{\Omega_{3}} \\
& \times \int_{0}^{\infty} \mathrm{d} x_{2} \int_{0}^{\infty} \mathrm{d} x_{3} x_{2}^{-2 m+1+2 i_{1}+1} x_{3}^{-2 m+1+1} \mathrm{e}^{-\frac{m}{\Omega_{1}}} \frac{x_{2}^{2} x_{3}^{2}-\frac{\kappa+1}{\Omega_{2}} x_{2}^{2}-\frac{1}{\Omega_{3}} x_{3}^{2}}{x_{2} x_{3}} \\
& \times\left(1+\frac{x^{2}}{\Omega_{1}} \frac{m}{\kappa+1}+\frac{x^{2}}{x_{2}^{2} x_{3}^{4}} \frac{\Omega_{3}}{\Omega_{1}} m\right)^{\frac{1}{2}} . \quad(17)
\end{aligned}
$$

The previous two-fold integral may be solved using the Laplace approximation theorem for the solution the twofold integrals [36], [37]:

$$
\int_{0}^{\infty} \mathrm{d} x_{2} \int_{0}^{\infty} \mathrm{d} x_{3}\left(x_{2}, x_{3}\right) \mathrm{e}^{\lambda f\left(x_{2}, x_{3}\right)}=\frac{\pi g\left(x_{20}, x_{30}\right)}{\lambda B\left(x_{20}, x_{30}\right)} \mathrm{e}^{\lambda f\left(x_{20}, x_{30}\right)},
$$

where $\mathrm{B}$ is the matrix:

$$
B\left(x_{20}, x_{30}\right)=\left|\begin{array}{ll}
\frac{\partial^{2} f\left(x_{20}, x_{30}\right)}{\partial x_{20}^{2}} & \frac{\partial^{2} f\left(x_{20}, x_{30}\right)}{\partial x_{20} \partial x_{30}} \\
\frac{\partial^{2} f\left(x_{20}, x_{30}\right)}{\partial x_{20} \partial x_{30}} & \frac{\partial^{2} f\left(x_{20}, x_{30}\right)}{\partial x_{30}^{2}}
\end{array}\right|,
$$

and $x_{20}$ and $x_{30}$ are solution of the equations:

$$
\frac{\partial f\left(x_{20}, x_{30}\right)}{\partial x_{20}}=0, \quad \frac{\partial f\left(x_{20}, x_{30}\right)}{\partial x_{30}}=0
$$

For considered case, it is:

$$
\begin{gathered}
g\left(x_{2}, x_{3}\right)=x_{2}^{-2 m+2 i_{1}+2} x_{3}^{-2 m+2} \\
\times\left(1+\frac{x^{2}}{x_{2}^{4} x_{3}^{2}} \frac{\Omega_{2}}{\Omega_{1}} \frac{m}{\kappa+1}+\frac{x^{2}}{x_{2}^{2} x_{3}^{4}} \frac{\Omega_{3}}{\Omega_{1}} m\right)^{\frac{1}{2}}, \\
f\left(x_{2}, x_{3}\right)=-\frac{m}{\Omega_{1}} \frac{x^{2}}{x_{2}^{2} x_{3}^{2}}-\frac{(\kappa+1)}{\Omega_{2}} x_{2}^{2}-\frac{1}{\Omega_{3}} x_{3}^{2}, \\
\frac{\partial f\left(x_{2}, x_{3}\right)}{\partial x_{2}}=\frac{2 m}{\Omega_{1}} \frac{x^{2}}{x_{2}^{3} x_{3}^{2}}-\frac{2(\kappa+1)}{\Omega_{2}} x_{2}, \\
\frac{\partial f\left(x_{2}, x_{3}\right)}{\partial x_{3}}=\frac{2 m}{\Omega_{1}} \frac{x^{2}}{x_{2}^{2} x_{3}^{3}}-\frac{2}{\Omega_{3}} x_{3},
\end{gathered}
$$

The solutions of the next two equations are $x_{20}$ and $x_{30}$ :

$$
\begin{gathered}
\frac{2 m}{\Omega_{1}} \frac{x^{2}}{x_{2}^{3} x_{3}^{2}}-\frac{2(\kappa+1)}{\Omega_{2}} x_{2}=0, \\
\frac{2 m}{\Omega_{1}} \frac{x^{2}}{x_{2}^{2} x_{3}^{3}}-\frac{2}{\Omega_{3}} x_{3}=0 .
\end{gathered}
$$

They should be introduced in Eq. (18) for solving two-fold integral from Eq. (17). In this manner LCR of the product of Nakagami- $m$, Rician and Rayleigh random variables will be obtained in a closed form.

\subsection{Numerical Examples and Discussion}

The level crossing rate of the product of Nakagami- $m$ random variable, Rician RV and Rayleigh RV is shown in the next few figures versus resulting signal $x$ for different values of fading parameters and signal powers.

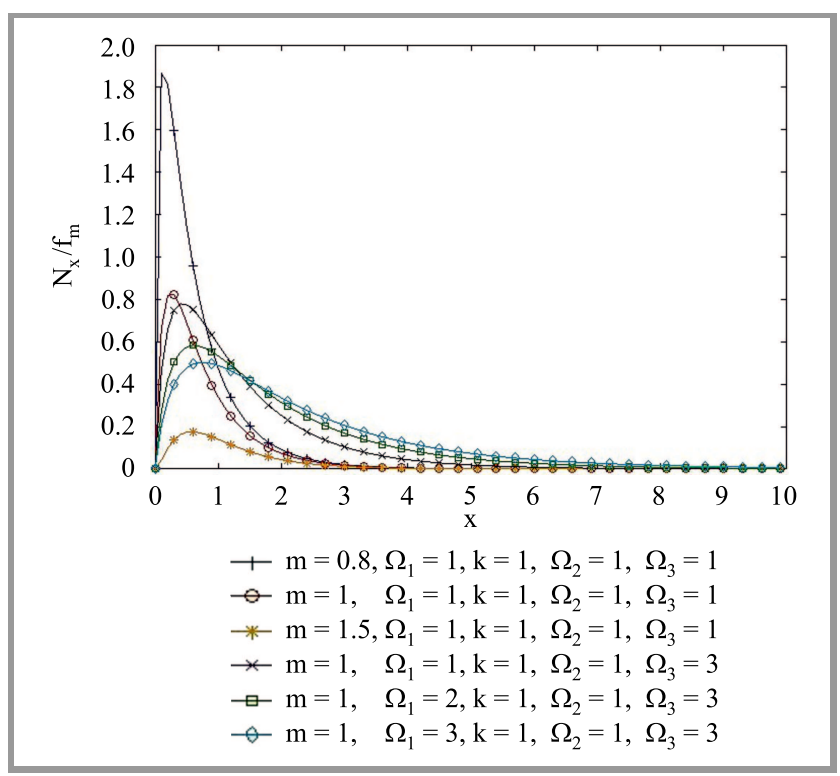

Fig. 1. LCR normalized by $f_{m}$ depending on signal envelope $x$ for various values of parameters $m$ and $\Omega_{1}$. 
Dependence of the LCR, normalized by $f_{m}$, on the resulting signal $x$, for various values of parameters $m$ and $\Omega_{1}$ is presented in Fig. 1. It is possible to notice that LCR increases for lower values of resulting signal and decreases for greater values of the resulting signal. All curves reach the maximum and start to decline. Lower values of the resulting signal have a greater impact on LCR. LCR increases for low values of Nakagami- $m$ small scale fading parameter $m$. The impact of resulting $x$ on LCR is larger for smaller magnitudes of parameter $m$. LCR is larger for smaller values of $m$.

From this picture, the influence of power $\Omega_{1}$ can also be observed. For low values of $x$, LCR increases with the reduction of power $\Omega_{1}$, but for bigger values of $x$, LCR increases along with the growth of power $\Omega_{1}$.

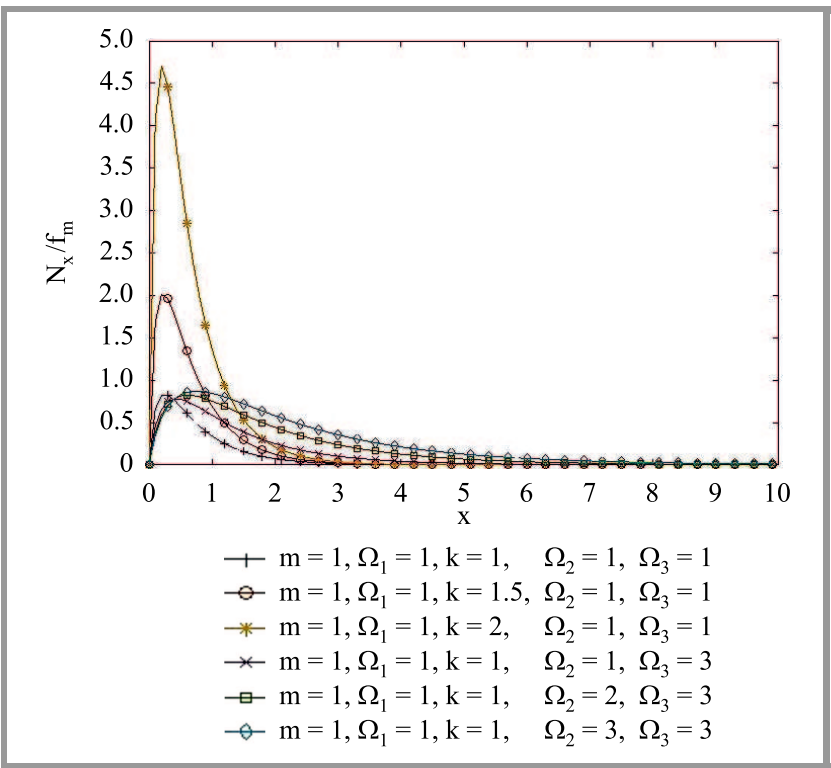

Fig. 2. LCR normalized by $f_{m}$ for different parameters $\kappa$ and $\Omega_{2}$.

Figure 2 shows the influence of the other two parameters: Rician factor $\kappa$ and signal power $\Omega_{2}$. LCR becomes bigger as the Rician factor $\kappa$ grows. The influence of $x$ on LCR is greater for lower values of Rician factor $\kappa$. The impact of Nakagami- $m$ fading parameter $m$ on LCR is higher for bigger values of Rician factor $\kappa$. From this figure, one can also see that LCR is larger for greater values of power $\Omega_{2}$. In Fig. 3 the impact of power $\Omega_{3}$ is shown. Based on the image, one may remark that LCR is higher for bigger values of $\Omega_{3}$ and low values of $x$. For higher values of $x, \mathrm{LCR}$ is greater for smaller $\Omega_{3}$. The small resulting signal $x$ exerts a greater impact on LCR.

The results obtained may be used to evaluate LCR of the product of Nakagami- $m$ and two Rayleigh RVs, LCR of Rician and two Rayleigh RVs, and LCR of the product of three Rayleigh (3* Rayleigh) RVs. This can be achieved because Nakagami- $m$ and Rician distributions are of the general variety. For the same reason, LCR of the product of three independent Rician RVs from [26] can be used for determination of LCR of the product of three Rayleigh $\mathrm{RVs}$, or the LCR of the product of two Rician RVs and

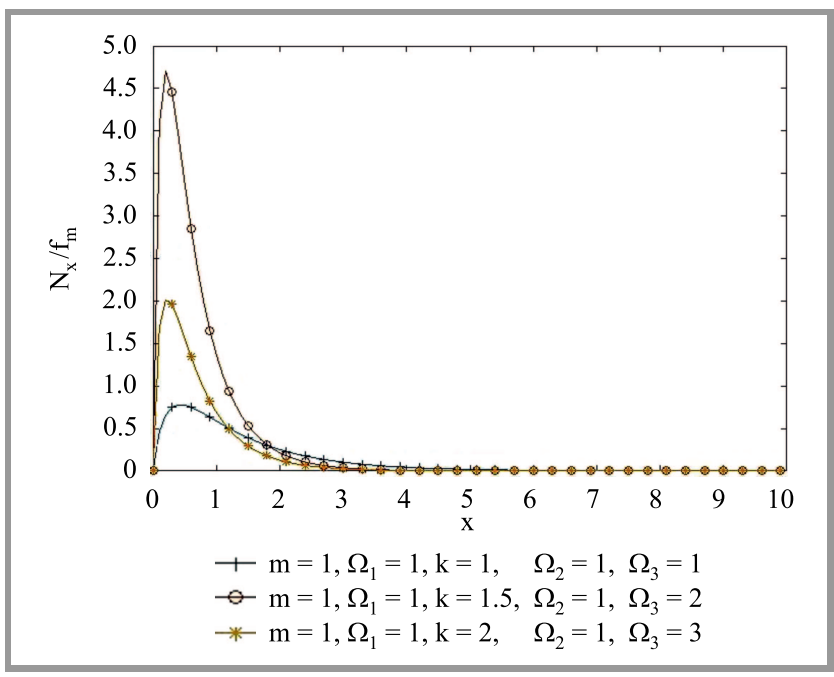

Fig. 3. LCR normalized by $f_{m}$ for various values of $\Omega_{3}$.

Rayleigh RV, or LCR of the product of Rician RV and two Rayleigh RVs, because Rayleigh distribution may be easily derived from Rician distribution for Rician factor $\kappa=0$.

If Nakagami fading severity parameter $m=\frac{1}{2}$, Nakagami distribution is reduced to unilateral (one-sided) Gaussian distribution. For $m=1$, Nakagami distribution reduces to Rayleigh distribution, and for $m>1$, Nakagami distribution is reduced to Rician distribution. The ratio between Rician factor $\kappa$ and parameter $m$ is [27], [38]:

$$
\kappa=\frac{\sqrt{m^{2}-m}}{m-\sqrt{m^{2}-m}}, \quad m>1 .
$$

On the other hand, for [31]:

$$
m=\frac{(\kappa+1)^{2}}{2(\kappa+1)},
$$

the distribution in Eq. (1) is approximately Rician fading with parameter $\kappa$. For $m=\infty$ we get an additive white Gaussian noise (AWGN) channel without fading. We see that as $m$ increases, fading decreases.

As the Nakagami distribution does not contain a Bessel function, it can get the close form solution more convenient than Rician distribution [30].

Thus, the Nakagami distribution may model Rayleigh distribution and Rician distribution, with certain restrictions [21]. Note that some empirical measurements support values of the $m$ parameter being equal to less than one, in which case the Nakagami fading causes a more severe performance degradation than Rayleigh fading.

\section{LCR of Product of Two Rician and Nakagami- $m$ Random Variables}

In the second example, presented in this section, Rician fading exists in the first two sections and Nakagami- $m$ fading is present in the third section. These results are applicable 
in analyzing the performance of multi-hop relay wireless telecommunications systems when the signal level is much higher than the noise level. In such a case, the noise level can be ignored. For that matter, the output signal is a product of as many random variables as there are sections in the relay system [38].

\subsection{Distribution of the Second Set of Random Variables}

Let random variables $x_{4}$ and $x_{5}$ have Rician distribution [22]:

$$
\begin{aligned}
& p_{x_{4}}\left(x_{4}\right)=\frac{2\left(\kappa_{1}+1\right)}{\Omega_{1}} \sum_{i_{2}=0}^{\infty}\left(\frac{\kappa_{1}\left(\kappa_{1}+1\right)}{\Omega_{1}}\right)^{i_{2}} \\
& \times \frac{1}{\left(i_{2} !\right)^{2}} x_{4}^{2 i_{2}+1} \mathrm{e}^{-\frac{\kappa_{1}+1}{\Omega_{1}} x_{4}^{2}}, \quad x_{4} \geq 0, \\
& p_{x_{5}}\left(x_{5}\right)=\frac{2\left(\kappa_{2}+1\right)}{\Omega_{2}} \sum_{i_{3}=0}^{\infty}\left(\frac{\kappa_{2}\left(\kappa_{2}+1\right)}{\Omega_{2}}\right)^{i_{3}} \\
& \times \frac{1}{\left(i_{3} !\right)^{2}} x_{5}^{2 i_{3}+1} \mathrm{e}^{-\frac{\kappa_{2}+1}{\Omega_{2}} x_{5}^{2}}, \quad x_{5} \geq 0,
\end{aligned}
$$

and let random variable $x_{6}$ have Nakagami- $m$ distribution [29]:

$p_{x_{6}}\left(x_{6}\right)=\frac{2}{\Gamma\left(m_{3}\right)}\left(\frac{m_{3}}{\Omega_{3}}\right)^{m_{3}} x_{3}^{2 m_{3}-1} \mathrm{e}^{-\frac{m_{3}}{\Omega_{3}} x_{6}^{2}}, x_{4} \geq 0, x_{6} \geq 0$,

where $\Omega_{i}, i=1,2,3$, are powers of RVs $x_{i}, i=4,5,6$, $\kappa_{1}$ and $\kappa_{2}$ are Rician factors for variables $x_{4}$ and $x_{5}$, and $m_{3}$ is Nakagami- $m$ fading severity parameter of RV $x_{6}$.

\subsection{Product of Three Random Variables}

Here, the random variable $x$ is defined as a product of $x_{i}$, $i=4,5,6$ :

$$
x=\prod_{i=4}^{6} x_{i} .
$$

The first time derivative of $x$ is:

$$
\dot{x}=\dot{x}_{4} x_{5} x_{6}+x_{4} \dot{x}_{5} x_{6}+x_{4} x_{5} \dot{x}_{6},
$$

the average value of $x$ is:

$$
\overline{\dot{x}}=\overline{\dot{x}}_{4} x_{5} x_{6}+x_{4} \overline{\dot{x}}_{5} x_{6}+x_{4} x_{5} \overline{\dot{x}}_{6}=0,
$$

because [29]

$$
\overline{\dot{x}}_{4}=\overline{\dot{x}}_{5}=\overline{\dot{x}}_{6}=0 \text {. }
$$

The variance of $\dot{x}$ is given by:

$$
\sigma_{\dot{x}}^{2}=x_{5}^{2} x_{6}^{2} \sigma_{\dot{x}_{4}}^{2}+x_{4}^{2} x_{6}^{2} \sigma_{\dot{x}_{5}}^{2}+x_{4}^{2} x_{5}^{2} \sigma_{\dot{x}_{6}}^{2},
$$

with:

$$
\begin{aligned}
& \sigma_{\dot{x}_{4}}^{2}=\pi f_{m}^{2} \frac{\Omega_{1}}{\kappa_{1}+1}, \\
& \sigma_{\dot{x}_{5}}^{2}=\pi f_{m}^{2} \frac{\Omega_{2}}{\kappa_{2}+1},
\end{aligned}
$$

$$
\sigma_{\dot{x}_{6}}^{2}=\pi f_{m}^{2} \frac{\Omega_{3}}{m_{3}} .
$$

After transformation of Eqs. (35)-(37) into Eq. (34), the variance is:

$$
\begin{aligned}
& \sigma_{\dot{x}}^{2}= \pi^{2} f_{m}^{2}\left(x_{5}^{2} x_{6}^{2} \frac{\Omega_{1}}{\kappa_{1}+1}+\frac{x^{2}}{x_{5}^{2}} \frac{\Omega_{2}}{\kappa_{2}+1}+\frac{x^{2}}{x_{6}^{2}} \frac{\Omega_{3}}{m_{3}}\right) \\
&=\pi^{2} f_{m}^{2} x_{5}^{2} x_{6}^{2} \frac{\Omega_{1}}{\kappa_{1}+1} \\
& \times\left(1+\frac{x^{2}}{x_{5}^{4} x_{6}^{2}} \frac{\Omega_{2}}{\kappa_{2}+1} \frac{\kappa_{1}+1}{\Omega_{1}}+\frac{x^{2}}{x_{5}^{2} x_{6}^{4}} \frac{\Omega_{3}}{m_{3}} \frac{\kappa_{1}+1}{\Omega_{1}}\right) .
\end{aligned}
$$

Joint PDF of $x, \dot{x}, x_{5}$ and $x_{6}$ is:

$$
\begin{aligned}
p_{x \dot{x} x_{5} x_{6}}\left(x \dot{x} x_{5} x_{6}\right)=p_{\dot{x}}(\dot{x} / & \left.x x_{5} x_{6}\right) \\
& \times p_{x}\left(x / x_{5} x_{6}\right) p_{x_{5}}\left(x_{5}\right) p_{x_{6}}\left(x_{6}\right),
\end{aligned}
$$

and joint PDF of $x$ and $\dot{x}$ :

$$
\begin{aligned}
& p_{x \dot{x}}(x \dot{x})=\int_{0}^{\infty} \mathrm{d} x_{5} \int_{0}^{\infty} \mathrm{d} x_{6} p_{x \dot{x} x_{5} x_{6}}\left(x \dot{x} x_{5} x_{6}\right) \\
& =\int_{0}^{\infty} \mathrm{d} x_{5} \int_{0}^{\infty} \mathrm{d} x_{6} p_{\dot{x} / x x_{5} x_{6}}\left(\dot{x} / x x_{5} x_{6}\right) p_{x}\left(x / x_{5} x_{6}\right) p_{x_{5}}\left(x_{5}\right) p_{x_{6}}\left(x_{6}\right),
\end{aligned}
$$

with:

$$
\begin{gathered}
p_{x}\left(x / x_{5} x_{6}\right)=\left|\frac{\mathrm{d} x_{4}}{\mathrm{~d} x}\right| p_{x_{4}}\left(\frac{x}{x_{5} x_{6}}\right), \\
\frac{\mathrm{d} x_{4}}{\mathrm{~d} x}=\frac{1}{x_{5} x_{6}} .
\end{gathered}
$$

The expression for $p_{x \dot{x}}(x \dot{x})$ in Eq. (40), after some replacements is:

$$
\begin{aligned}
p_{x \dot{x}}(x \dot{x})=\int_{0}^{\infty} \mathrm{d} x_{5} \int_{0}^{\infty} \mathrm{d} x_{6} p_{\dot{x}}\left(\dot{x} / x x_{5} x_{6}\right) \\
\quad \times \frac{1}{x_{5} x_{6}} p_{x_{4}}\left(\frac{x}{x_{5} x_{6}}\right) p_{x_{5}}\left(x_{5}\right) p_{x_{6}}\left(x_{6}\right) .
\end{aligned}
$$

\subsection{LCR of Product of the Second Set of Random Variables}

Level crossing rate of $x$ is defined by Eq. (16) [39]. LCR of product $x$ from Eq. (30), with $p_{x \dot{x}}(x \dot{x})$ from Eq. (43), is:

$$
\begin{aligned}
N_{x}=\int_{0}^{\infty} \mathrm{d} x_{5} \int_{0}^{\infty} \mathrm{d} x_{6} & \left(\int_{0}^{\infty} \mathrm{d} \dot{x} \dot{x} p_{\dot{x}}\left(\dot{x} / x x_{5} x_{6}\right)\right. \\
& \left.\times \frac{1}{x_{5} x_{6}} p_{x_{4}}\left(\frac{x}{x_{5} x_{6}}\right) p_{x_{5}}\left(x_{5}\right) p_{x_{6}}\left(x_{6}\right)\right) .
\end{aligned}
$$

After introducing Eqs. (27)-(29) and Eq. (38) into Eq. (44), we obtain LCR as: 


$$
\begin{aligned}
& N_{x}=\frac{1}{\sqrt{2 \pi}} \pi f_{m} \frac{\Omega_{1}^{\frac{1}{2}}}{\left(\kappa_{1}+1\right)^{\frac{1}{2}}} x^{2 i_{1}+1} \frac{2\left(\kappa_{1}+1\right)}{\Omega_{1}} \sum_{i_{2}=0}^{\infty}\left(\frac{\kappa_{1}\left(\kappa_{1}+1\right)}{\Omega_{1}}\right)^{i_{2}} \\
& \times \frac{1}{\left(i_{2} !\right)^{2}} \frac{2\left(\kappa_{2}+1\right)}{\Omega_{2}} \sum_{i_{3}=0}^{\infty}\left(\frac{\kappa_{2}\left(\kappa_{2}+1\right)}{\Omega_{2}}\right)^{i_{3}} \frac{1}{\left(i_{3} !\right)^{2}} \frac{2}{\Gamma\left(m_{3}\right)} \\
& \times\left(\frac{m_{3}}{\Omega_{3}}\right)^{m_{3}} \int_{0}^{\infty} \mathrm{d} x_{5} \int_{0}^{\infty} \mathrm{d} x_{6} x_{5}^{-2 i_{2}-1+2 i_{3}+1} x_{6}^{-2 i_{2}-1+2 m_{3}-1} \\
& \times \mathrm{e}^{-\frac{\kappa_{1}+1}{\Omega_{1}} \frac{x^{2}}{x_{5}^{2} x_{6}^{2}}-\frac{\kappa_{2}+1}{\Omega_{2}} x_{5}^{2}-\frac{m_{3}}{\Omega_{3}} x_{6}^{2}} \\
& \times\left(1+\frac{x^{2}}{x_{5}^{4} x_{6}^{2}} \frac{\Omega_{2}}{\kappa_{2}+1} \frac{\kappa_{1}+1}{\Omega_{1}}+\frac{x^{2}}{x_{5}^{2} x_{6}^{4}} \frac{\Omega_{3}}{m_{3}} \frac{\kappa_{1}+1}{\Omega_{1}}\right)^{\frac{1}{2}} .
\end{aligned}
$$

Now we need to use the Laplace approximation theorem for the solution of double integrals, defined in Eqs. (18)-(20), for $x_{5}$ and $x_{6}$, and $x_{50}$ and $x_{60}$ as solutions [39], to solve last integrals in Eq. (45).

For this case the following is valid:

$$
\begin{gathered}
g\left(x_{5}, x_{6}\right)=x_{5}^{-2 i_{2}+2 i_{3}} x_{6}^{-2 i_{2}+2 m_{3}-2} \\
\times\left(1+\frac{x^{2}}{x_{5}^{4} x_{6}^{2}} \frac{\Omega_{2}}{\kappa_{2}+1} \frac{\kappa_{1}+1}{\Omega_{1}}+\frac{x^{2}}{x_{5}^{2} x_{6}^{4}} \frac{\Omega_{3}}{m_{3}} \frac{\kappa_{1}+1}{\Omega_{1}}\right)^{\frac{1}{2}}, \\
f\left(x_{5}, x_{6}\right)=-\frac{\kappa_{1}+1}{\Omega_{1}} \frac{x^{2}}{x_{5}^{2} x_{6}^{2}}-\frac{\kappa_{2}+1}{\Omega_{2}} x_{5}^{2}-\frac{m_{3}}{\Omega_{3}} x_{6}^{2}, \\
\frac{\partial f\left(x_{5}, x_{6}\right)}{\partial x_{6}}=\frac{2\left(\kappa_{1}+1\right)}{\Omega_{1}} \frac{x^{2}}{x_{5}^{3} x_{6}^{2}}-\frac{2\left(\kappa_{2}+1\right)}{\Omega_{2}} x_{5}, \\
\frac{\partial f\left(x_{5}, x_{6}\right)}{\partial x_{6}}=\frac{2\left(\kappa_{1}+1\right)}{\Omega_{1}} \frac{x^{2}}{x_{5}^{2} x_{6}^{3}}-\frac{2 m_{3}}{\Omega_{3}} x_{6} .
\end{gathered}
$$

\subsection{Numerical Examples and Discussion}

Level crossing rate of the product of two Rician random variables and a Nakagami- $m$ random variable is calculated and shown in the next few figures. The influence that Rician factors $\kappa_{1}$ and $\kappa_{2}$, Nakagami- $m$ fading severity parameter $m_{3}$, Rician multipath fading powers $\Omega_{1}$ and $\Omega_{2}$, and Nakagami- $m$ fading power $\Omega_{3}$ exert on LCR is discussed.

LCR, normalized by $f_{m}$, depending on the signal envelope $x$, is presented in Fig. 4, for different values of Rician factor $\kappa_{1}$ and Rician fading power $\Omega_{1}$. It is obvious from the picture that LCR achieves the maximum for small values of signal envelope $x$, and starts to decrease for higher values of $x$. So, it is evident that the impact of the signal envelope on LCR is bigger for small values of the signal envelope. It is also visible from this figure that LCR increases along with the increase in Rician factor $\kappa_{1}$ and in power $\Omega_{1}$. It is known that system performance is better for smaller values of LCR.

In Fig. 5, the normalized LCR is shown versus signal envelope for different values of parameters $\kappa_{1}$ and $\Omega_{2}$. It is

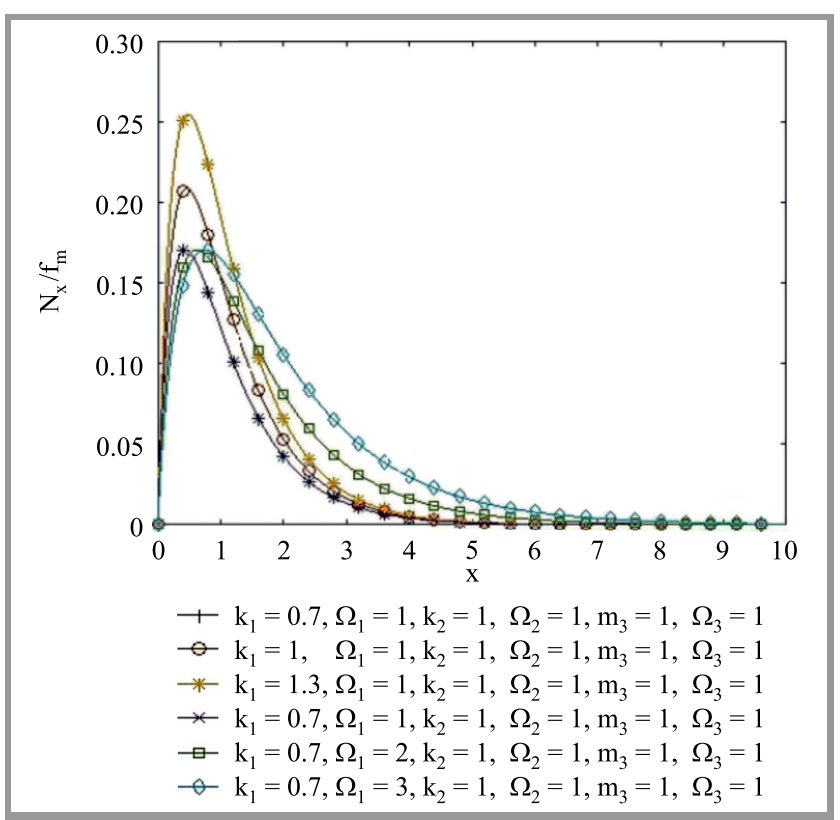

Fig. 4. LCR normalized by $f_{m}$ versus signal envelope $x$ for various values of parameters $\kappa_{1}$ and $\Omega_{1}$.

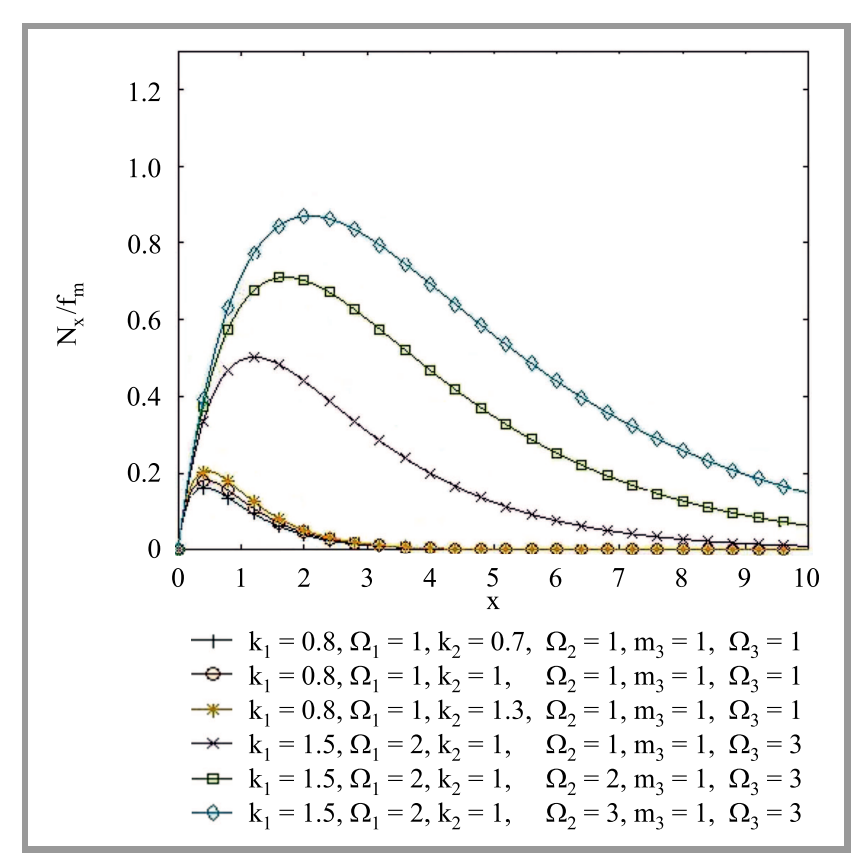

Fig. 5. LCR normalized by $f_{m}$ versus signal envelope $x$ for several values of parameters $\kappa_{2}$ and $\Omega_{2}$.

possible to see from this figure that when $\kappa_{1}$ grows, LCR increases as well, but the increase is insignificant. On the other hand, with the increase in $\Omega_{2}$, LCR increases visibly, the curves become wider and the maximums move towards higher values of the signal envelope $x$.

The last figure, Fig. 6, presents the LCR, normalized by $f_{m}$, depending on signal envelope for various values of Nakagami- $m$ fading severity parameter $m_{3}$, and Nakagami- $m$ fading power $\Omega_{3}$. It can be noticed that LCR grows with an increase in fading power $\Omega_{3}$ and with a reduction in the Nakagami- $m$ fading parameter $m_{3}$. 


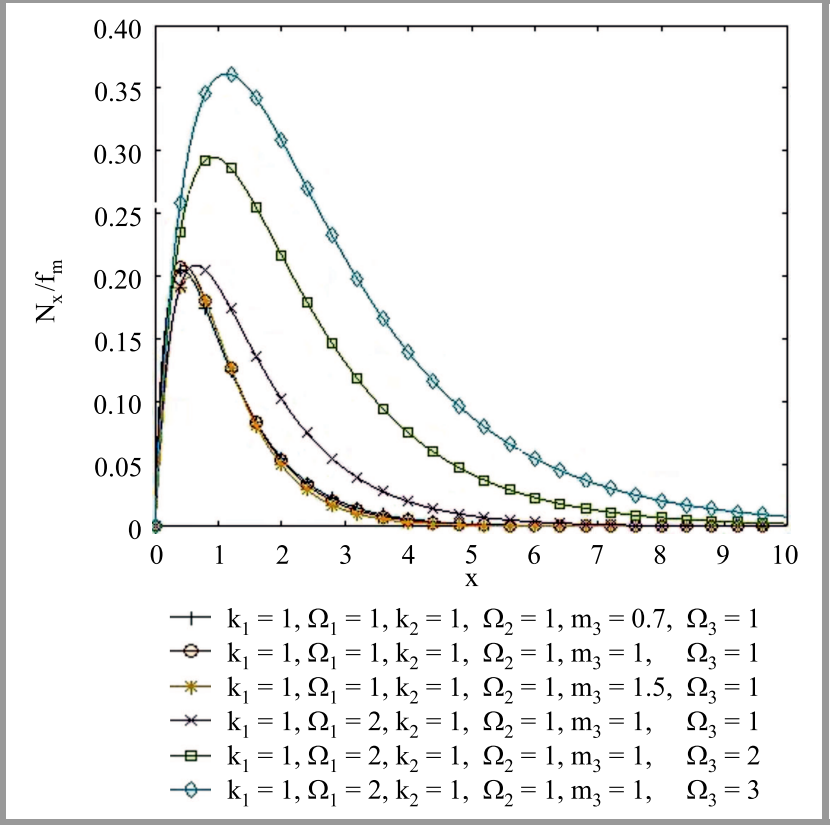

Fig. 6. LCR normalized by $f_{m}$ versus signal envelope for different values of parameters $m_{3}$ and $\Omega_{3}$.

\section{Conclusion}

The product of RVs is applied in multiple relay channels in the presence of composite fading. In this work, we focused on a wireless relay communications channel with three sections, where the product of three RVs describes the amplitude at the output of the cascaded fading channel with three sections. A closed form LCR has been calculated for that channel. The formula obtained has been checked for different values of fading and power parameters.

The results are valuable for scientists and system designers dealing with fading models for different wireless channels. It is possible to verify the proposed distribution of the products of other fading amplitudes by measuring parameters in real wireless relay channels in the presence of multipath fading, and also due to the fact that Nakagami- $m$ and Rician distributions are of the general variety. By entering adequate values of fading parameters, other fading distributions in the individual sections may be obtained.

\section{Acknowledgments}

This work was partially supported under projects TR-33035 and III-44006 of the Ministry of Education, Science and Technological Development of Republic of Serbia.

\section{References}

[1] Y. Chen, G. K. Karagiannidis, Hao Lu, and Ning Cao, "Novel approximations to the statistics of products of independent random variables and their applications in wireless communications", IEEE Trans. on Veh. Technol., vol. 61, no. 2, 2012, pp. 443-454 (doi: 10.1109/TVT.2011.2178441).
[2] S. Nadarajaha and D. K. Dey, "On the product and ratio of t random variables", Appl. Mathem. Lett., vol. 19, no. 1, pp. 45-55, 2006 (doi: 10.1016/j.aml.2005.01.004).

[3] E. Mekić , N. Sekulović, M. Bandjur, M. Stefanović, and P. Spalević, "The distribution of ratio of random variable and product of two random variables and its application in performance analysis of multi-hop relaying communications over fading channels", Przeglad Elektrotechniczny (Electrical Review), vol. 88, no. 7a, pp. 133-137, 2012.

[4] D. Krstic, M. Stefanovic, V. Milenkovic, and Dj. Bandjur, "Level crossing rate of ratio of product of two $\alpha-\mathrm{k}-\mu$ random variables and $\alpha$-k- $\mu$ random variable", WSEAS Trans. on Commun., vol. 13, no. 1 , pp. 622-630, 2014.

[5] D. Krstic, I. Romdhani, M. B. Y. Masadeh, S. Minic, G. Petkovic, and P. Milacic, "Level crossing rate of ratio of product of two k$\mathrm{u}$ random variables and Nakagami-m random variable", IEEE Int. Conf. on Comp. and Inform. Technol.; Ubiquitous Comput. and Commun.; Depend., Autonom. and Sec. Computi.; Perv. Intellig. and Comput., Liverpool, UK, 2015 (doi: 10.1109/CIT/IUCC/DASC/PICOM.2015.244).

[6] N. Zlatanov, Z. Hadzi-Velkov, and G. K. Karagiannidis, "Level crossing rate and average fade duration of the double Nakagami- $m$ random process and application in MIMO keyhole fading channels", IEEE Commun. Lett., vol. 12, no. 11, pp. 822-824, 2008 (doi: 10.1109/LCOMM.2008.081058).

[7] J. D. Donahue, "Products and quotients of random variables and their applications", ARL 64-115, Aerospace Research Laboratories, Wright-Patterson Air Force Base, Ohio, The Martin Company, Denver, Colorado, July 1964 [Online]. Available: https://apps.dtic.mil/dtic/tr/fulltext/u2/603667.pdf

[8] M. D. Springer and W. E. Thompson, "The distribution of products of independent random variables", SIAM J. on Appl. Mathem., vol. 14, no. 3, pp. 511-526, 1966 (doi: 10.1137/0114046).

[9] M. D. Springer and W. E. Thompson, "The distribution of products of beta, gamma and Gaussian random variables", SIAM J. on Appl. Mathem., vol. 18, no 4, pp. 721-737, 1970 (doi: 10.1137/0118065).

[10] Z. A. Lomnicki, "On the distribution of products of random variables", J. of the Royal Statist. Soc. Series B (Methodological), vol. 29, no. 3, pp. 513-524, 1967.

[11] A. G. Glen, L. M. Leemis, and J. H. Drew, "Computing the distribution of the product of two continuous random variables", Comput. Statist. and Data Anal., vol. 44, no. 3, pp. 451-464, 2004 (doi: 10.1016/S0167-9473(02)00234-7).

[12] T. S. Glickman and F. Xu, "The distribution of the product of two triangular random variables", Statist. \& Probab. Lett., vol. 78, no. 16, pp. 2821-2826, 2008 (doi: 0.1016/j.spl.2008.03.031).

[13] G. K. Karagiannidis, N. C. Sagias, and P. T. Mathiopoulos, "N*Nakagami: a novel stochastic model for cascaded fading channels", IEEE Trans. Commun., vol. 55, no. 8, pp. 1453-1458, 2007 (doi: 10.1109/TCOMM.2007.902497).

[14] Z. Zheng, L. Wei, J. Hamalainen, and O. Tirkkonen, "Approximation to distribution of product of random variables using orthogonal polynomials for lognormal density", IEEE Commun. Lett., vol. 16, no. 12 , pp. $2028-2031,2012$ (doi: 10.1109/LCOMM.2012.101712.122141).

[15] E. J. Leonardo and M. D. Yacoub, "Statistics of the product of arbitrary $\alpha-\mu$ variates with applications", in Proc. 25th Int. Symp. on Pers., Indoor and Mob. Radio Commun. PIMRC 2014, Washington, DC, USA, 2014, pp. 73-76 (doi: 10.1109/PIMRC.2014.7136135).

[16] Z. Stojanac, D. Suess, and M. Kliesch, "On products of Gaussian random variables", arXiv:1711.10516 [math.PR], 2018.

[17] N. Bhargav et al., "On the product of two $\kappa-\mu$ random variables and its application to double and composite fading channels", IEEE Trans. on Wirel. Commun., vol. 17, no. 4, pp. 2457-2470, 2018 (doi: 10.1109/TWC.2018.2796562).

[18] D. H. Pavlovic et al., "Statistics for ratios of Rayleigh, Rician, Nakagami- $m$, and Weibull distributed random variables", Mathem. Problems in Engin., vol. 2013, Article ID 252804 (doi: 10.1155/2013/252804). 
[19] M. Shakil and B. M. Golam Kibria, "On the product of Maxwell and Rice random variables", J. of Modern Appl. Statist. Meth., vol. 6 , no. 1 , Article 19 , pp. 212-218, 2007 (doi: $10.22237 /$ jmasm/1177993080) [Online]. Available: http://digitalcommons.wayne.edu/jmasm/vol6/iss1/19

[20] K. Pearson, "The problem of the random walk", Nature, vol. 72, p. 318, 1905 (doi: 10.1038/072294b0).

[21] M. Nakagami, "The $m$-distribution: A general formula of intensity distribution of rapid fading", in Statistical Methods in Radio Wave Propagation: Proceedings of a Symposium held June 18-20, 1958, W. C. Hoffman, Ed. New York: Pergamon Press, 1960, pp. 3-36 (doi: 10.1016/b978-0-08-009306-2.50005-4).

[22] S. O. Rice, "Mathematical analysis of random noise", Bell Syst. Technic. J., vol. 24, no. 1, pp. 46-156, 1945 (doi: 10.1002/j.1538-7305.1945.tb00453.x).

[23] D. Chizhik, G. J. Foschini, M. J. Gans, and R. A. Valenzuela, "Keyholes, correlations, and capacities of multielement transmit and receive antennas", IEEE Trans. on Wirel. Commun., vol. 1, no. 2, pp. 361-368, 2002 (doi: 10.1109/7693.994830).

[24] T. Taniguchi, Y. Karasawa, and M. Tsuruta, "An analysis method of double fading MIMO channels including LOS environments", in Proc. of the IEEE 19th Int. Symp. Pers., Indoor Mob. Radio Commun., Cannes, France, 2008, pp. 1-5 (doi: 10.1109/PIMRC.2008.4699512).

[25] D. Krstic, M. Stefanovic, and P. Nikolić, "Level crossing rate of product of Nakagami- $m$ random variable, Rician random variable and Rayleigh random variable", ICTF 2018, IEICE Information and Communication Technology Forum, July 2018, Graz, Austria.

[26] D. Krstic, M. Stefanovic, M. M. B. Yaseen, S. Aljawarneh, and P. Nikolić, "Statistics of the product of three Rician random processes with application", in Proc. 1st Int. Conf. on Data Sci., Elearn. and Inform. Syst. DATA'18, Madrid, Spain, 2018 (doi: 10.1145/3279996.3280015).

[27] G. L. Stüber, Principles of Mobile Communication, 2nd ed. Norwell, MA, USA: Kluwer, 2001 (ISBN: 0792379985).

[28] P. M. Shankar, Fading and Shadowing in Wireless Systems. New York Dordrecht Heidelberg London: Springer, 2012 (doi: 10.1007/978-1-4614-0367-8).

[29] S. Panic, M. Stefanovic, J. Anastasov, and P. Spalevic Fading and Interference Mitigation in Wireless Communications. Boca Raton, USA: CRC Press, 2013 (ISBN: 9781466508415).

[30] D. Shen, Y. Cui, A. Zhang, Y. Yang, and K. Wu, "A simple simulation method for Nakagami fading channel", in Proc. Int. Conf. on Microw. and Millim. Wave Technol., Chengdu, China, 2010 (doi:10.1109/icmmt.2010.5525262).

[31] A. Goldsmith, Wireless Communications. Stanford University, 2004 (ISBN: 9780521837163).

[32] S. O. Rice, "Statistical properties of a sine wave plus random noise", Bell Syst. Tech. J., vol. 27, no. 1, pp. 109-157, 1948 (doi:10.1002/j.1538-7305.1948.tb01334.x).

[33] A. Mitić, D. Milović, M. Jakovljević, A. Panajotović, "Second order statistics of signal in Nakagami - lognormal fading channels with selection combining", in XIII Telekomunikacioni forum TELFOR 2005, Beograd, Serbia, 2005 [in Serbian].

[34] M. D. Yacoub, J. E. V. Bautista, and L. Guerra de Rezende Guedes, "On higher order statistics of the Nakagami- $m$ distribution", IEEE Trans. on Veh. Technol., vol. 48, no. 3, pp. 790-794, 1999 (doi: 10.1109/25.764995).

[35] T. S. Rappaport, Wireless Communications: Principles and Practice, 2nd ed. Upper Saddle River, N.J.: Prentice Hall, 2002 (ISBN: 0130422320).

[36] M. Abramowitz and I. A. Stegun, Handbook of Mathematical Functions. National Bureau of Standards, 1964, reprinted Dover Publications, 1965 (ISBN: 9780486612720).

[37] J. L. Lopez and P. J. Pagola, "A simplification of the Laplace method for double integrals. Application to the second Appell function*", Electron. Trans. on Num. Analysis, vol. 30, pp. 224-236, 2008 (ISSN: 1068-9613).
[38] N. C. Karmakar, Ed., Handbook of Smart Antennas for RFID Systems. Wiley, 2010 (doi: 10.1002/9780470872178, ISSN: 1068-9613).

[39] Z. Cao and Y. D. Yao, "Definition and derivation of level crossing rate and average fade duration in an interference-limited environment", in Proc. IEEE 54th Veh. Technol. Conf. VTC Fall 2001, Atlantic City, N.J., USA, 2001 (doi: 10.1109/VTC.2001.956470).

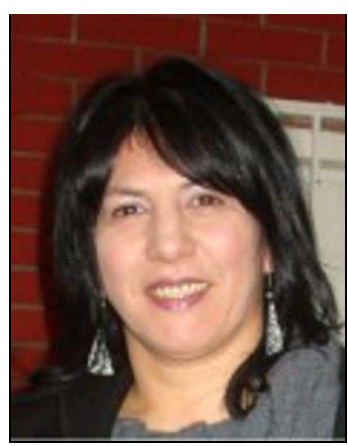

Dragana S. Krstic received her B.Sc., M.Sc. and Ph.D. degrees in Electrical Engineering from the Faculty of Electronic Engineering, University of Niš, Serbia in 1990, 1998. and 2006, respectively. She has been working at the Faculty of Electronic Engineering, University of Niš, since 1990. Her fields of interest include telecommunications theory, optical communication systems, as well as wireless, mobile and satellite communication systems. As an author or co-author, she wrote about 260 scientific research papers, of which about 60 have been printed in international journals, several in national journals, and close to 140 have been referred to international symposia and conferences. Dr. Krstic has held more plenary and keynote lectures, panels and tutorials, by invitation, at international conferences and some faculties. She is also a member of several international journals and a reviewer for many. Also, she is a'member of the technical program committees for nearly a hundred conferences and a reviewer for about 120 conferences.

(iD) https://orcid.org/0000-0002-2579-3911

E-mail: dragana.krstic@elfak.ni.ac.rs

Faculty of Electronic Engineering

University of Niš

Niš, Serbia

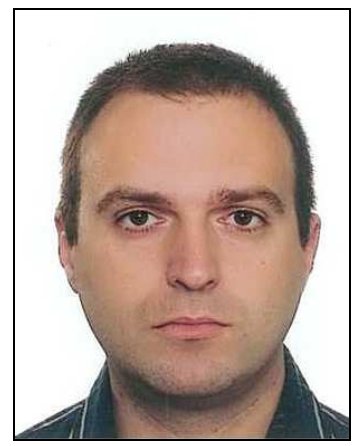

Petar B. Nikolić graduated from the Faculty of Electronic Engineering, University of Niš, Serbia, and received his M.Sc. and Ph.D. degrees in 2008 and 2016 , respectively. He is working for a company of Tigar Tyres, Pirot. His main research interest is connected with the wireless communication systems. He has written or coauthored a considerable number of papers, has published in renowned journals and conferences proceedings.

E-mail: nikpetar@gmail.com

TigarTyres

Pirot, Serbia 


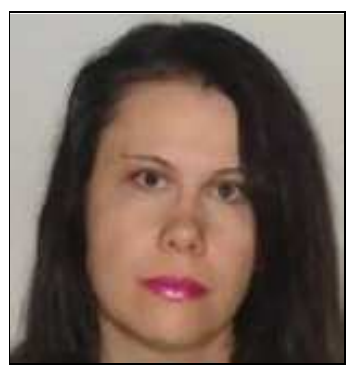

Danijela A. Aleksić received the B.Sc. degree in Electronics and Telecommunications Engineering from the Faculty of Electronic Engineering, University of Niš, Serbia in 2001, and M.Sc. degree in the field of electromagnetics at the Faculty of Technical Sciences in Cacak, Serbia, in 2010. Her research interests are statistical models of wave propagation in urban environments and diversity techniques for reducing the influence of fading on the performance of wireless systems. She is working at College of Applied Technical Sciences, Niš since 2002. She was a lab technician, then a teaching assistant. Currently, she works as a lecturer. Previous field of interest are sensors and transducers. She has written or co-authored of some papers, published in eminent journals and conferences proceedings.

E-mail: danijela.aleksic@vtsnis.edu.rs

College of Applied Technical Sciences

Niš, Serbia

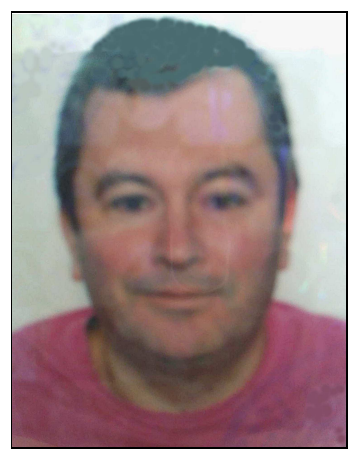

Dragan Z. Vučković received the M.Sc. degree in the field of safety and health from the Faculty of Occupational Safety, University of Niš, Serbia in 2004, and B.Sc. degree in the field of electrical engineering and computing at the College of Applied Technical Sciences, Serbia in 2006. His research interest are storage of electronic waste and waste management on railways. He worked at Joint Stock Company for freigh trailway transport "Srbija
Cargo" a.d. since 1998. He was a leading engineer for safety and health. Currently he works as a chief coordinator for work and fire protection. He has written or co-authored of some papers, published in eminent journals and conferences proceedings. He is currently on doctoral studies at the Faculty of Economics and Engineering Management, Department of Ecology, University Business Academy, Novi Sad, Serbia.

E-mail: dvdvucko@gmail.com

Faculty of Economics and Engineering Managment University Business Academy

Novi Sad, Serbia

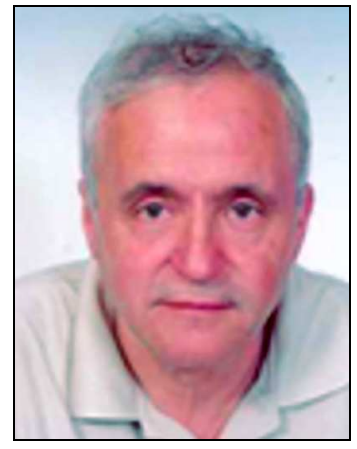

Mihajlo C. Stefanović received the B.Sc., M.Sc. and Ph.D. degrees in Electrical Engineering from the Faculty of Electronic Engineering, Department of Telecommunications, University of Nis, Serbia, in 1971, 1976 and 1979, respectively. His primary research interests are statistical communication theory, optical and wireless communications. He has written or coauthored a great number of journal publications. He has written five monographs, too. He was a mentor to hundreds of graduates, for dozens of master's theses and doctoral dissertations, and many times a member of commissions for the defense of such works. Now, Dr. Stefanović is a retired professor at the Faculty of Electronic Engineering in Niš.

(i) https://orcid.org/0000-0002-8063-8981

E-mail: mihajlo.stefanovic@elfak.ni.ac.rs

Faculty of Electronic Engineering

University of Niš

Niš, Serbia 